\title{
Die Hauptrichtungen der finnischen Wortartforschung
}

1. Die Wortartkategorien und ihr System sind das Ergebnis einer historischen Entwicklung. Daher können die Wortartgruppen sogar verwandter Sprachen grosse Unterschiede voneinander aufweisen. Ein Wortartensystem, das die Entwicklung einer gegebenen Sprache und deren heutigen Zustand widerspiegelt, wäre also unmöglich ohne weiteres auf eine andere Sprache anzuwenden.

Trotz alledem glauben wir, dass die wortarttheoretischen Systematisierungen und die wortartgeschichtlichen Forschungen der Finnen in mancher Hinsicht auch der ungarischen Wortartforschung zum Nutzen gereichen können. Erstens: die verhältnismässig reiche und vielseitige wortarttheoretische Fachliteratur der Finnen kann - bloss mit ihren Gesichtspunkten und Methoden - auf die Hebung unserer ziemlich ämlichen Wortartforschung anregend wirken. Wohl sind die Wortartkategorien der finnischen Sprache nicht identisch mit denen der unsrigen. Vom Ungarischen abweichend hat das Finnische zum Beispiel keinen Artikel und kein Verbalpräfix. Jas finnische Nomen besitzt keine prädizierende Kraft, während eine solche eine seit altersher bekannte Tatsache im lingarischen ist. Eine anffallende Eigentümlichkeit des finnischen Eigenschaftsworts ist, dass dassolbe als Attribut mit seinem Hauptwort im Kasus und Numerus kongruiert, während im Ungarischen das adjektivische Attribut sich nicht nach dem Hauptglied richtet. Unzweifelhaft kann aber der Hinweis auf gerade solche Unterschiede die besonderen Eigentümlichkeiten der Wortarthategorien unseror Muttersprache besser als alles andere beleuchten. Es gibt im Ungarischen und im Finnischen auch identische oder ähnliche Wortarterscheinungen. 
Das sind entweder aus der finnisch-uyrischen, bzw. uralischen Vorzeit ererbte uralte Eigentümlichkeiten, oder auf Grund gemeinsamer Vorgeschichte des Systems entstandene parallele sondersprachliche Entwicklungen. Ob wir nun die bewahrten Altertümlichkeiten, oder die parallelen Entwicklungen in Betracht ziehen, die Kenntnis der Entsprechungen der verwandten Sprachen wird die muttersprachlichen Kategorien zum tieferen Bewusstsein bringen.

Natürlich können wir es hier nicht unternehmen, die Geschichte der finnischen Wortartforschung und deren heutigen Stand in allen ihren Einzelheiten darzustellen. Wir lassen die Vorführung der Ergebnisse solcher - übrigens grundlegender .-. Studien weg, die zwar die Erscheinungen einer bestimmten Wortart untersuchten, doch nicht in erster Linie vom Gesichtspunkte der Wortart ${ }^{1}$. In der vorliegenden Arbeit möchte ich nur über die Hauptrichtungen der finnischen Wortartforschungen und deren wichtigste Ergebnisse Rechenschaft geben.

\section{Das traditionelle siystem}

2. Das traditionelle Wortartsystem wird durch die Schulgrammatiken, u.d. E. N. SETÄLё's Grammatiken vertreten.

Setälä unterscheidet in seiner finnischen Grammatik ${ }^{2}$ vom formellen Gesichtspunkt, d.i. auf Grund der Wortbiegung drei Wortarten: 1. Nomina, 2. Verba, 3. Partikeln; auf Grund der Bedeutung aber hält er neun Wortarten in Evidenz: 1. Hauptwörter, 2. Eigenschaftswörter, 3. Zahlwörter, 4. Fürwörter, 5. Zeitwörter, 6. Bestimmungswörter, 7. Postpositionen und Präpositionen, 8. Bindewörter, 9. Ausrufewörter. Durch Zusammenfassung der beiden (resichtspunkte aber entsteht folgendes Wortartsystem:

1 Solche sind u.a. das grundlegende Buch von Aug. Ahlqvist Suomen Kielen Rakennus I 1877, von dem ein grosser Teil sich mit den Nomina beschäftigt; Arvid Genetz's Dissertation über die Partikeln: Suomen partikkelimuodot 1890; die Pronomenlehre von Heikk Ijansuv: Itämerensuomalaisten kielten pronominioppia 1922.

2 E. N. Setäl Ä Suomen kielioppi. 193012. S. 45-47. 
A. Nomina

1. Hauptwörter

a. konkrete (Gattungsnamen, Eigennamen)

b. abstrakte

2. Eigenschaftswörter

3. Zahlwörter

4. Fürwörter

B. Zeitwörter

C. Partikeln

1. Bestimmungswörter

2. Postpositionen und Präpositionen

3. Bindewörter

4. Ausrufewörter

Setäläs Wortartsystem zeigt die Fehler und Vorzüge des traditionellen Systems. Sein Hauptfehler ist die Inkonsequenz, die auf die Mehrdeutigkeit des Aufteilungsprinzips zurückzuführen ist. Setälä bringt schon wenigstens den morphologischen und den semantischen Gesichtspunkt zur Geltung, doch hebt er die syntaktische Grundlage der Einteilung nicht hervor. Der Vorzug dieses Systems hingegen ist dic Natürlichkeit gegenüber jeder gekünstelten Einteilung aus e i $n$ e m Gesichtspunkt, also die Rücksichtnahme auf die sprachliche Wirklichkeit.

\section{Reformbestrebungen in der Schule}

3. Im Jahre 1906 wurde in Finnland oin grammatischer Ausschuss gegründet und mit der Aufgabe betraut, einen Entwurf zur Vereinheitlichung der grammatischen Terminologie, der grammatischen Begriffe und Definitionen für die Schule auszuarbeiten. Der Ausschuss wurde im Jahre 1915 mit seinem Bericht $^{1}$ fertig, dessen Grundgedanken in erster Linie die des zweiten Präsidenten des Ausschusses, E. N. Setäläs widerspiegeln.

Im Bericht nimmt der Teil über die Wortarten einen wichtigen Platz ein (S. 20-33, 94-101). Neu ist vor allem die Unterscheidung zwischen zwei Wortbegriffen nach Noreess Lehre:

1 Komiteanmietintö N:o 6. 1915. 
1. "sana" (Wort), 2. "sane" (Wortform). "Sane" ist in der Rede der kleinste Teil mit besonderer Bedeutung. Es vereinigt in sich eine grammatikalische und eine lexikalische Bedeutung. So bezeichnet z.B. die Wortform karhun (im Ausdruck karhun pesä 'des Bären Nest') einerseits das 'Tier mit dem Namen karhu 'Bär' (das ist die lexikalische Bedeutung, die mit dem Stamm verbunden ist), andererseits den Umstand, dass es sich um einen einzigen Bären handelt und es sich um einen Bären als Besitzer handelt (das ist die grammatische Bedeutung, die durch die Endung gekennzeichnet ist). Es gibt auch Wortformen, in deren morphologischer Struktur man die grammatische Bedeutung von der lexikalischen nicht trennen kann, z.B. nyt 'jetzt'. Während "sane" oder "Wortform" ein Bestandteil des grammatischen Systems ist, bildet "sanla", d.i. "Wort" einen T'eil des Wortschatzes. Das Wort hat also nur lexikalische Bedeutung. Ein Wort, das mehrere Wortformen besitzt, pflegt man abwandlungsfähig (flektierbar) zu nennen (z.B. heronen 'Pferd', laulau 'singen'). Wenn hingegen ein Wort nur eine gebräuchliche Form hat, nennen wir es unflektierbar (z.B. nyt 'jetzt', pikku 'klein'). Die flektierbaren Wörter sind wieder von zwei Arten: 1. Nomini, die nur nominale Formen haben und 2. Verba, die sowohl über verbale als auch nominale Formen verfügen. Auf diese Weise entsteht ein derartiges System der Wortarten:

\section{I, flektierbare Wörter \\ 1. Nomina \\ 2. Verba \\ II. unflektierbare Wörter}

Gleichfalls nach Norees ${ }^{2}$ teilt der grammatische Ausschuss die Wörter in zwei grosse Gruppen ein: 1. Nennwörter und 2 . Zeigewörter. ${ }^{3}$ Die Nennwörter benennen Vorstellungen (Gegenstands-, Eigenschafts-, Zahl-, Handlungs-, Umstandsrorstellun-

1 Für den letzteren beantragte Setälä selbst in einem Vortrag von 1915 das Fachwort "vaihen, s. JSFOu. 43 S. 14. Vgl. Istvíx PAPP MNy. 49 S. 359 f. und MNy. 51 S. 290 f.

2 Adolf Noreen Vårt språk II. 1904. S. 63 ff́.

3 Zur wissenschaftgeschichtlichen Vorgeschichte der Aufteilıng vgl. PaAvo Ravila Vir. 1953 S. 46 
gren), z.B. hevonen 'Pferd', rushea 'braun', kolme 'drei'. Die Zeigewörter oder pronominalen Wörter weisen hingegen nur auf gewisse Vorstellungen hin, z.B. minä 'ich', sellainen 'solcher', sinne 'dahin'.

U̇brigens hat der Ausschuss im grossen und ganzen die traditionelle Wortartengruppierung angenommen. El weicht davon bloss darin ab, dass er nach Noreens Beispiel die herkömmliche Gruppe der Bindewörter in zwei Gruppen spaltet, und einerseits dic beiordnenden Bindewörter als Konjunktionen als besondere Wortart qualifiziert, andererseits die unterordnenden Bindewörter als subjunktionen als besondere Wortart betrachtet. So tritt an Stelle der älteren Neunereinteilung im Antrag cine Zehnereinteilung: 1. Hauptwörter, 2. Eigenschaftswörter, 3. Zahlwörter, 4. Fürwörter, 5. Zeitwörter, 6. Bestimmungswörter, 7. Präpositionen und Postpositionen, 8. Konjunktionen, 9. Subjunktionen, 10. Ausrufewörter. Ein neues Moment bedeutet auch der Umstand, dass der Ausschuss in den Definitionen der einzelnen Wortartenkategorien neben dem morphologischen und semantischen Gesichtspunkt auch den syntaktischen bewusst geltend zu machen suchte.

\section{Eine Einzelfrage}

4. Schon der grammatische Ausschuss hat in seinem Bericht darauf hingewiesen (a.a.O. S. 99), dass die Klärung des Bereiches und der Funktion der Bestimmungswörter eine der schwierigsten Aufgaben der Wortarttheorie ist. Diese Frage hat Enıк Ahisan in seiner Arbeit über Adverbien untersucht ${ }^{1}$.

Nach Ahlman kann die logisch-semantische Definition der Kategorie der Bestimmungswörter zu keinem Ergebnis führen, da es schwirrig sein würde solche begriffliche Zeichen zu finden, die bei jedem Bestimmungswort als gemeinsamer Zug eine Rolle spielten. Die Bestimmungswörter drücken angeblich irgendeinen "Umstand" aus; dieses Bedeutungsmoment ist aber so unbe-

1 Erik Ahlman Adverbeista. Vir. 1933 S. 137-159. Dasselbe deutsch: Über Adverbien. Studia Fennica 3 S. 19-44. 
stimmt und verschwommen, dass es ummöglich ist, darauf irgendeine Definition zu gründen. Auch sonst wäre es ein hoffnungsloses Unternehmen, die einzelnen Wortarten auf lediglich semantischem (trunde abzugrenzen. Man pflegt z.B. sagen, dass das Adjektiv eine Eigenschaft, das Verb eine Handlung oder pinen Zustand ansdrücke. Wenn das wirklich so wäre, müssten wir das Wort laumeus 'Schönheit' als Adjektiv qualifizieren, und juoksu 'Liaf' als Verb; doch wissen wir, dass beide Hauptwörter sind.

Mehr Erfolg verspricht der syntaktische Gesichtspunkt. Nach diesem steht das Bestimmungswort als Ergänzung des Verbs oder Adjektivs. Diese Definition ist allerdings in vielen Fällen guiltig. Doch gibt es auch Ausnahmen. Wir wissen, dass nicht nu' Bestimmungswörter, sondern auch Hauptwörter als Ergäuzung des Verbs stehen kömnen. ferner dass Bestimmungswörter nicht nur ein Verb oder ein Adjektiv, sondern auch ein Hauptwort bestimmen können, z.B. tuo poika tuolla 'jener Knabe dort'.

Nun bleibt noch die morphologische Definition. Als wichtigsten formalen Zug des Bestimmungswortes pflegt man dessen Indeklinierbarkeit zu erwähnen. Doch gibt es auch Bestimmungswörter, die klar eine Kasusform zeigen, z.B. sisüllä 'drinnen' (Adessiv), ullirma 'draussen' (Essiv), täältä 'von hier' (Ablativ). Ausserdem kann man viele Bestimmungswörter steigern, z.B. launiisti 'schön', kauniimmin 'schöner'. Daraus folgt, dass die Indeklinierbarkeit nicht zum wichtigsten Kennzeichen des Bestimmungswort gehört, wenigstens nicht im Finnischen.

Es gibt aber eine andere formale Eigenschaft, die zu dieser Rolle zu passen scheint. Das ist die Erscheinung der Kongruenz. Danach sind die Bestimmungswörter solche Wörter, welche mit ihrem Hauptglied in Numerus und Kasus nicht kongruieren, und mit denen auch ihre Ergänzungen formal nicht kongruieren. Die erste Hälfte der Regel ist geeignet, die Bestimmungswörter von den Adjektiven abzugrenzen. So ist das Wort hyvin im Ausdruck hyvin myöhään 'sehr (eigentl. 'gut') spät' Bestimmungswort, weil es mit seinem Hauptglied nicht kongruiert; hingegen Adjektiv im Ausdruck hyvin aikomuksin 'mit guter 
Absicht', weil hier die Kongruenz schon durchgeführt ist. Die zweite Hälfte der Regel kann zur Abgrenzung der Bestimmungswörter von den Hauptwörtern dienen. So z.B. ist mielellään im Ausdruck hycin mielellään 'sehr gern' Bestimmungswort, doch wäre es in der Konstruktion hycällä mielellään 'in guter Stimmung' ein Hauptwort.

5. Der Kreis der Bestimmungswörter ist aber nach mehreren Seiten offen, die Grenzlinie gegen andere Wortarten mehrfach schwankend. Das ist auch ganz natürlich, da doch auch hier - wie anderswo - - Ibergangswortkategorien vorkommen.

Ein besonderes Problem bedenten im Fimnischen vor allem die dort vorkommenden undeklinierbaren Eigenschaftswörter, die formal mit ihrem Hauptglied also nicht kongruieren. z.B. aika 'ziemlich gross', ensi 'd. erste', pikku 'klein'. Vom Cresichtspunkt der Kongruenz aus sind diese Eigenschaftswörter bestimmungswortartig. Bezeichnenderweise unterscheiden sie sich nicht nur durch diese eine Eigenschaft, sondern auch durch andere von den übrigen Eigenschaftswörtern: man kimn sie nicht steigern, sie können keine Crradbestimmung zu sich nehmen (man kann nicht sagen: liian pikku), sie können nicht als Prädikat steh'n (man kinn nicht sagen: se on piliku), rinige sind auch als Bestimmungswörter des (Arades gebräuchlich (z.B. ailia suuri 'recht gross'). All das weist darauf hin, dass die undeklinierbaren Eigenschaftswörter der finnischen Sprache wahrlich eine Ċbrgangskategorie zwischen Eigenschafts- und Bestimmungswörteru sind.

Eine Schwierigkeit bedeuten anderseits die Fürwörter. Die substantivischen Fürwörter können nämlich kein adjektivisches Attribut zu sich nehmen. wie die Hauptwörter im allgemeinen. Die substantivischen Fürwörter verhalten sich demgemäss wie die Bestimmungswörter. Auch einzelne Formen der adjektivischen Fürwörter reqieren einen Partitiv ohne Kongruenz, z.B. tällä tapaa 'auf diese Weise'. Es ist auch kein Zufall, dass sich aus Fürwörtern oft Bestimmungswörter entwickelt haben, z.B. tuolla 'dort', missä 'wo', näin 'so, auf diese Weise'. Das alles zeigt, dass die Fürwörter von mehreren Gesichtspunkten aus İbrualngskategorien sind. 
Dasselbe können wir von den Gruppen der Postpositionen, der Präpositionen und der Konjunktionen sagen. Ihr mit den Bestimmungswörtern gemeinsamer Zug ist das Fehlen der Kongruenz. Ihre Abgrenzung von den Bestimmungswörtern ist nur auf syntaktischer Grundlage möglich. Wir können nämlich sagen, dass die Bestimmungswörter einer einseitigen Ergänzung bedürfen, die Postpositionen, Präpositionen und Konjunktionen hingegen einer zweiseitigen. So ist das Wort ympärillä in der Fügung ympärillä oli paljon ihmisiä 'viele Leute waren ringsumher' ein Bestimmungswort, im Satze aber miehen ympärillä oli paljon ihmisiä 'viele Leute waren um den Mann' eine Postposition.

6. Ahlman teilt die Bestimmungswörter auf Grund ihrer Bedeutung und hauptsächlich ihrer syntaktischen Eigentümlichkeiten in drei Gruppen ein: 1. inhaltsvolle Bestimmungswörter, welche Ort, Zeit, Art und Weise, und Zustand bedeuten, z.B. kotona 'zu Hause', eilen 'gestern', helposti 'leicht', hereillä 'wach'; 2. Bestimmungswörter des Grades, d.h. solche, die den Grad, die Abstufung einer Eigenschaft bezeichnen, z.13. hyvin 'sehr', noin 'so', tavattoman 'ausserordentlich'; 3. Modifizierende, d.h. solche, die das Mass der Gültigkeit, Einschränkung oder Ausdehnung, Hervorhebung, also im allgemeinen ein irgendwie subjektives Verhältnis bezeichnen, z.B. ainakin 'wenigstens', vain 'nur', vielä 'noch'.

Der Verfasser analysiert ausführlich das syntaktische Verhalten der einzelnen Arten der Bestimmungswörter: als was für Satzteile sie stehen können, als Ergänzung welcher Wörter, was für Ergänzungen sie annehmen können und mit was für Wörtern dieselben nebenordnende Verbindungen bilden können.

7. Ahlman hat in der Untersuchung der Bestimmungswörter vor allem Gesichtspunkte struktureller Art angeführt. Die Erforschung der verwickelten Verhältnisse des Sprachbaus zeitigte die Lehre, dass es ausser den bestimmten Wortartbildungen auch Übergangswortkategorien gibt, deren Funktionen sich in keiner bestimmten Richtung kristallisiert haben. Die Untersuchung der strukturellen Eigentümlichkeiten der 
sprache war auf diese Weise geeignet, auch die Linien der Wortartentwicklung aufzuzeigen. Dass die Bestimmungswörter der heutigen finnischen sprache Übergänge zu mehreren anderen Wortartgruppen zeigen, und dass die Untergruppen der Bestimmungswörter auch miteinander in entwicklungsgeschichtlicher Verbindung stehen: das zum Bewusstsein gebracht zu haben, ist das wichtigste Ergebnis der strukturellen Analyse.

\section{Von der Seile der Theorie her}

8. Matti Airila betrachtete die Erscheinungen der finnischen Sprache gern von theoretischer Höhe. Er näherte sich auch der Frage des finnischen Wortartensystems von der Theorie, der allgemeinen Sprachwissenschaft her. Sein Buch über die Wortarten erschien als ein Band seiner einleitenden Arbeit über die Sprachtheorie. ${ }^{1}$ Darin fasste er auch die Ergebnisse seiner älteren wortarttheoretischen Arbeiten zusammen ${ }^{2}$.

Airila übernahm im Grunde die traditionelle Gruppierung der Wortarten. Nur hier und da versuchte er die auffallenden Inkonsequenzen der Wortarttradition zu tilgen. In seinem System führte er zuerst semantische Gesichtspunkte an und nahm syntaktische Gesichtspunkte nur in zwciter Linie in Anspruch. Der rein formale Gesichtspunkt trat dagegen bei ihm vollständig in den Hintergrund.

Auf diese Weise stellte er folgendes Wortartensystem zusammen:

I. Ausrufewörter (Interjektionen)

II. Redewörter

1. Zeigewörter (Fürwörter)

2. Nennwörter (expressive Wörter)

A. inhaltsvolle Wörter

a. Hauptwörter (Gattungsnamen, Eigennamen)

b. Eigenschaftswörter

c. Bestimmungswörter

d. Zeitwörter

1 Matti Airila Johdatusta kielen teoriaan III, Sanaluokat. 1952.

2 Eine solche ist z.B. seine Studie über die Fürwörter: Pronominit. Vir. 1940 S. $301-314$. 
B. Formenwörter
a. Zahlwörter
b. Situativa
c. gradbezeichnende und Schätzungswörter
d. Postpositionen
e. Bindewörter
f. Modifizierer

In der Gruppe der Formenwörter haben die Zahlwörter und die Situativa objektiven Charakter, die Postpositionen, Bindewörter und Modifizierer subjektiven, die gradbezeichnenden und Schätzungswörter abrr teilweise objektiven. teilweise subjektiven.

9. Die Wörter zerfielen auf Crrund ihrer Bedeutung primär in obige Wortartgruppen. 1)ie so ausgebildeten Gruppen passten für bestimmte syntaktische Rollen. In der Urzeit der Sprache entsprachen die Denkkategorien und die Wortartgruppen im grossen und ganzen einander. So erschien z.B. die logischf Kategorie des Gegenstandes immer in der Form des Hauptwortes, das im Satze Subjekt- oder andere ähnliche Rollen spielen konnte. Mit der Entwicklung des Denkens parallel aber äusserte sich immer mehr auch in der sprache das Bedürfnis, die Eigenschaften, Geschehnisse und ander Denkkategorien zum Gegenstand einer eben solchen besonderen Anschanung machen zu können wie den konkreten Gegenstandsinhalt des Hauptwortes. Solchen Beweggründen wird man es zuschreiben können, dass man auch auf Eigenschaften und Geschehnisse manchmal die morphologischen und syntaktischen Eigentümlichkeiten übertrug, d.h. auch mit Eigenschafts-, Geschehnisund anderen Begriffen so umzugehen begann, als wenn sie Gegenstände, Realitäten wären. Diese Erscheinung nannte Airila Verwandlung ("muuntuminen") und schrieb in der Ausbildung des Systems der Wortarten gerade dieser Gesetzmässigkeit die grösste Bedeutung zu. Die Wortartenverwandlung d.h. die kategorielle Verschiebung - besteht darin, dass derselbe Bedeutungsinhalt in der Form verschiedener Wortarten erscheinen kann, bzw. dass die Wörter ohne die geringste Änderung des Bedeutungsinhaltes aus einer Wortartengruppe 
in eine andere übertragbar sind. Die zwei Hauptkennzeichen der Wortartenumwandlung sind: die Stabilität des Bedeutungsinhaltes und die Veränderlichkeit der syntaktischen Rolle.

Die Mittel der Umwandlung sind mehrerlei. Hierher gehört, dass wir das Wort bloss durch Anwendung von Endungen und Zeichen geeignet zu einer neuen syntaktischen Rolle machen. In anderen Fällen erreichen wir dasselbe Resultat durch Ableitungssilben. Von den Ableitungssilben kommen aber natürlich nur diejenigen in Betracht, die zugleich den Charakter der Wortart verändern, z.B. juoksen 'ich laufe' -- juoksu 'Lauf'. Als Umwandlung kann man von einem gewissen Gesichtspunkt auch den Umstand betrachten, dass ein Wort zugleich zu zwei Wortartkategorien gehört, z.B. zum Nomen und Verb zugleich (z.B. uskn 'Glaube' - uskoa 'glauben').

Im Anschluss an die Umwandlung ist die wichtigste Aufgabe die Klärung, wann wir rom identischen Bedeutungsinhalt der zu verschiedenen Kategorien gehörenden Wörter sprechen können. Airila versucht diese Frage mit folgendem Beispiel zu beleuchten. In den Wörtern punainen 'rot', punaisuus 'Röte', punoittaa 'rötlich leuchten' findet man kein solches Bedeutungsmoment, das nicht schon im Stamm puna 'Röte' vorhanden wäre. Das Hauptwort punaisuus bezeichnet die Eigenschalt eines Gegenstandes ebenso wie das Eigenschaftswort punainen, der Unterschied besteht nur darin, dass Ersteres die Eigenschaft vom konkreten Gegenstand abstrahiert, vorselbständigt als Objekt einer besonderen Anschauung vor uns stellt. Das ist aber bloss der kategorielle Unterschied zwischen Hauptwort und Eigenschaftswort, der den Bedeutungsinhalt selbst, den konkreten Sinn nicht berührt. Auch das Verb punoitlaa bedeutet nichts anderes als 'rot sein'. Was in einem Fall sprachlich als Eigenschaft erscheint (punainen, punaisuus), steht im anderen als Geschehnis vor uns (punoittaa). Das beruht aber nicht allf dem Unterschied des Bedeutungsinhaltes, sondern bloss auf dem Wortartunterschied zwischen Eigenschaftswort und Zeitwort. Hingegen gehören nicht zu den Wortartumwandlungen jene Fälle, bei denen irgendeine Ableitungssilbe dem Wort ein neues Bedeutungsmoment verleiht, z.B. metsä 'Wald' -- metsästä̈̈ 'jagen', hopea 'Silber' -- hopeoida 'versilbern'. 
10. Airilas Wortartensystem schliesst sich in mehreren Punkten organisch an die vorangehenden Versuche der Wortarttheorien an.

Er folgte Noreen und dem Beispiel des finnischen grammatischen Ausschusses unter anderem darin, dass er die Unterscheidung zwischen Zeigewörtern und Nennwörtern in sein semantisches System der Wörter einfügte. Den Kategoriecharakter des Wortes kann der Bedeutungsinhalt selbst bestimmen: auf dieser Grundlage können wir z.B. von Gegenstandswörtern (Hauptwörtern) und Eigenschaftswörtern (Adjektiven) sprechen. Den Wortartcharakter bestimmt aber auch dic Bedeutungsfunktion, d.h. die Art und Weise, in der irgendein Wort einen bestimmten Bedeutungsinhalt erweckt. So kommen die funktionalen Wortarten zustande. Wenn wir aber den Gesichtspunkt der Bedeutungsfunktion folgerichtig zu durchdenken versuchen, dann müssen wir neben die Fürwörter und Nennwörter als Drittes die Eigennamen aufnehmen. Airila sah zwar, dass die Bedeutungsfunktion des Eigennamens sich mit der des Fürwortes in mehreren Punkten berührt, doch hat er die systematischen schlussfolgerungen aus dieser Erkenntnis nicht gezogen.

Als Airilas wichtigste Neuerung können wir betrachten, dass er die traditionelle Gruppe der Bestimmungswörter in vier selbständige Wortarten aufteilte. Als Bestimmungswörter behielt er in seinem System nur die mit modaler Bedeutung, während er aus dem traditionellen Kreis der Bestimmungswörter die Situationswörter oder Situativa (die Ort, Zeit und Zustand bezeichnenden Bestimmungen), ferner die gradbezeichnenden und Schätzungswörter (die den Grad, die Abstufung der Eigenschaft und die Schätzung bezeichnen), endlich die Modifizierwörter (d.h. solche mit betonender oder mildernder Bedeutung) heraushob. Airila tritt im Endergebnis in Ahlmans Fussstapfen. Der Unterschied ist nur der, dass das, was bei Ahlman als Unterart der einheitlichen Bestimmungswortgruppe gilt, bei Airila als selbständige wortartliche Kategorie behandelt wird. Dazu kommt, dass er auch Ahlmans Gruppe der bedeutungsreichen Bestimmungswörter entzweigeschnitten hat. Er betrachtet nur die modalen als Bestimmungswörter und nennt diejenigen, die Ort, Zeit und Zustand bezeichnen, Situativa. 
So wurden aus den drei Ahlmanschen Bestimmungswörtergruppen bei Airila vier besondere Wortartenkategorien.

Airilas Verfahren, dass er einerseits die modalen Bestimmungswörter als besondere Kategorie hervorhebt, anderseits die Ort, Zeit und Zustand bedeutenden als Situativwörter betrachtet, erscheint uns als unbegründet. Wir können unserseits z.B. zwischen nopeasti 'schnell' "Bestimmungswort" und kaukana 'fern' "Situativwort" weder in Bedeutung, noch im Satzwert einen wesentlichen Unterschied feststellen. In der Bedeutung der beiden ist ein Inhaltsmoment enthalten (bei dem einen die Eigenschaft 'schnell', beim andern der Zustand 'fern') und ein formales, vergleichendes Moment (dort ein modales, hier ein Ortsverhältnis). Anderseits können beide im Satz mit selbständigem Satzteilwert als Bestimmung stehen. Das alles weist diarauf hin, dass sowohl das "Bestimmungsw ort», als auch das "Situativwort" auf gleiche Weise Übergangskategorien zwischen den bedeutungsreichen und Formenwörtern sind, anderseits aber zwischen den Wörtern mit Satzteilwert und solchen mit Verhältnisbezeichnung stehen.

Auch können wir nicht gutheissen, dass Airila die traditionelle Wortartgruppe der Postpositionen und Präpositionen aufteilte: die Ort, Zeit, Zustand bezeichnenden Wortarten wies er in den Bereich der Situativwörter, während diejenigen, die Ursach? oder Ziel ausdrücken, auch weiterhin in der Kategorie der Postpositionen blieben. Darin stimmen wir ihm bei, dass Ort. Zeit und Zustand ein konkreteres Verhältnis bedeuten als Ursache oder Ziel, doch darf man nicht vergessen, dass die Zeit und auch der Zustand abstrakter ist als das Ortsverhältnis. Die so gezogene Grenze zwischen »konkret» und "abstrakt», wie wir sie bei Airila finden, kann daher weder philosophisch noch vor der naiven Denkart standhalten und kann nicht rechtfertigen, diss man z.B. kanssa 'zusammen' (isän kanssa 'mit dem Vater') und tähden 'wegen' (perheeni tähden 'wegen meiner Familie') anf Grund des Bedentungsunterschiedes in zwei besondere Wortartgruppen einordnen soll. Richtig ist. dass die einzelnen Mitglieder der traditionellen Postpositionskategorie sowohl selbständig als auch unselbständig gebraucht werden können (z.B. hän asuu kirkon lähellä 'er wohnt in 
der Nähe der Kirche' - hän asuu lähellä 'er wohnt in der Nähe'); andere wieder können nur unselbständig stehen (z.B. perheeni tähden 'wegen meiner Familie'). Auch sonst kommt es vor, dass ein Wort ohne jegliche formale Änderung aus einer Wortartkategorie in eine andere übertritt. Es liegt darin also gar nichts besonderes, dass z.B. das Wort lähellä manchmal als Bestimmungswort (Airila: "Situativwort"), manchmal als Postposition steht. Das berechtigt aber noch nicht dazu, das Wort lähellä darum als "Situativwort" gegenüber den "Postpositionen" nach der Art tähden zu qualifizieren.

Airilas auffallendste Neuerung ist, dass er die Gesetzmässigkeit der Wortartenumwandlung (d.i. Kategorieverschiebung) folgerichtig und organisch in sein Wortartensystem einzubauen trachtete. Dass die verschicdenen Sprachen einen bestimmten Bedeutungsinhalt aus einer Wortartkategorie in eine andere zu versetzen in verschicdenem Masse fähig sind, haben die Forscher schon früh beobachtet und sie versuchten, die Rolle dieser Erscheinung in Denken und im sprachlichen System zu klären. U.a. hat schon Wilhelm Wundt über die kategorielle Verschiebung der Begriffe gesprochen ${ }^{1}$, Albert Sechenaye über die Übertragung, üher die Erscheinung der Transposition ${ }^{2}$. Englische Grammatiken heben die Erscheinung der sog. Konversion hervor. In der ungarischen und finnisch-ugrischen Sprachwissenschaft pflegt man von Nomen-Verba zu sprechen und vom Umschlagen der Wortkategorie, bzw. von Übergangswortkategorien. ${ }^{3}$

Ein grosses Verdienst der Wortartentheorie Airilas ist, diese von mehreren Seiten erkannte Erscheinung durchforscht und für die Systematisierung der Wortarten nutzbar gemacht zu haben. Die im Lichte der Umwandlung betrachteten Wortartenkategorien verlieren ihre Steifheit, das Wortartensystem wird dynamisch. Airilas Unternehmen konnte freilich nicht in

1 W. Wundt Logik I. Allgemeine Logik und Erkenntnistheorie. S. 113.

2 Albert Sechenaye Essai sur la structure logique de la phrase. 1936. S. 103.

3 Vgl. Gyula Laziczius NyK 51 S. 14 ff.; József Techert (Tompa) MNy. 38 S. 254 ff. 
jeder Hinsicht gelingen. Sind doch die sprachlichen Kategorien so vielseitig, so sehr im Zustande der Umwandlung begriffen, dass man eben diese Veränderlichkeit der Wortartenkategorien nur sehr schwer in ein folgerichtiges System bringen kann.

Auch ist es die Frage, ob es so einen Wortartumschlag, eine "Umwandlung» gibt, die den Sinn des Wortes, seinen Bedeutungsinhalt unberührt lässt und einfach nur seine morphologische Bildung und syntaktische Rolle verändert. Airila selbst weist an einer Stelle (S. 46-47) in Gegensatz zu Anton MarTy darauf hin, dass die Kategorie des Verbs auch in dessen Bedeutung sich von der Wortartgruppe des Nomens, bzw. des Adjektivs unterscheidet. Es kommt zwar vor, dass auch das Adjektiv einen Zustand ausdrückt wie das Verb, doch bezeichnet das Adjektiv den dauernden Charakter des Zustands, während das Verb das wechselnde Wesen des Zustands bedeutet, z.B. viheriä 'grün' - viheriöidä 'grünen'. Zur Bedeutung des Verbs gehört daher auch der Aspekt der Zeit. Demgemäss sehen wir darin einen Widerspruch, dass Airila an einer anderen Stelle (S. 70) darüber spricht, dass man angeblich im Adjektiv punainen 'rot' und im Verb punoittaa 'rötlich leuchten' kein solches Bedeutungsmoment auffinden kann, das im Grundwort puna 'Röte' nicht schon vorhanden wäre.

Airila macht zwischen den Bildungssuffixen, die eine kategorielle Verschiebung verursachen, einen Unterschied von dem Gesichtspunkte, ob sie dem Grundwort eine zusätzliche Bedeutung verleihen oder nicht, und betrachtet nur die letzteren als sprachliche Mittel der Wortartumwandlung. Nach ihm lassen z.B. solche Bildungen wie puna(inen): punoittaa oder kala 'Fisch': kalainen 'mit Fischen' den Bedeutungsinhalt des Grundwortes unberührt, sie verursachen bloss eine kategorielle Verschiebung. Demgegenüber hat sich in Bildungen wie hopea 'Silber': hopeoida 'versilbern' oder kala 'Fisch': kalastaa 'fischen' nicht nur die Wortkategorie, sondern auch der Bedeutungsinhalt verändert. Letztere gehören daher nach Airila nicht zum Bereich der Wortartumwandlung. Wir müssen gestehen, dass wir unsererseits zwischen den bedeutungsverwandelnden Rollen der behandelten zweierlei Bildungsarten keinen grundlegenden Unterschied zu entdecken vermögen. Das Verb punoittaa 'rot 
sein, rötlich leuchten' bedeutet soviel wie 'es scheint rot', zum Bedeutungsinhalt des Grundwortes 'rot' ist also nicht nur die allgemeine kategorielle Bedeutung des Verbs hinzugetreten, sondern auch die spezielle Verbalbedeutung 'es erscheint als etwas'. Gerade so wie im Falle von hopeoida 'versilbern', das eigentlich soviel bedeutet wie 'mit Silber überziehen' oder 'silberfarbig machen'. Ähnlich steht es mit Bildungen wie kalainen 'mit Fischen' und kalastaa 'fischen'. Das erstere knüpft nämlich an die Bedeutung kala 'Fisch' noch die adjektivische. Bedeutung 'etwas habend, mit etwas versehen, reich an etwas', das zweite verbindet mit derselben Grundbedeutung noch diejenige des Verbs 'etwas fangen, sammeln'. Wir können überhaupt nicht einsehen, warum die erstere zusätzliche Bedeutung inhaltlich gleich Null wäre, das letztere aber - wie Airila meint - positiv.

Auffallend ist, dass die Zahlwörter in Airilas System nicht zu den inhaltsvollen, sondern zu den Formwörtern gehören. Zweifelsohne kennzeichnet die Gruppe der Zahlwörter eine gewisse inhaltliche Leere, das ist nach unserer Meinung aber bloss mit der abstrakten Bedeutung verbunden. Man weiss, dass die heutigen abstrakten Zahlen sich aus konkreter Bedeutung entwickelt haben. Ausserdem findet man das Erogebnis des abstrahierenden Verfahrens auch bei andern Wortarten. So hat sich bei den Hauptwörtern über die mit älterer konkreter Bedeutung eine jüngere Schicht mit abstrakter Bedeutung gelegt. Wie wir die Wörter mit abstrakter Bedeutung - wegen ihrer Abstraktheit -- aus der Wortartkategorie der Hauptwörter nicht herausheben. so kann man auch die Zahlwörtor - wegen ihres abstrakten Bedeutungsinhalts - aus der Gruppe der inhaltsvollen Wörter nicht herausheben. ¿̈brigens ist bekannt, dass die Zahlwörter im Finnischen (d.i. die Grundzahlwörter) nach ihrem morphologischen und syntaktischen Verhalten teilweise substantivischen, teilweise adjektivischen Charakter haben ${ }^{1}$. Wenn wir die Sache ausschliesslich von der semantischen Seite betrachten, können wir sagen, dass das Zahlwort mit dem Eigen-

1 Vgl. Lauri Hakelinfon Suomen kielen rakenne ja kehitỵs I S. 66; Paavo Ravila Vir. 1953 S. 43. 
schaftswort nahe Verwandtschaft aufweist. Denn beide Wortarten bezeichnen rine kigenschaft: das Eigenschaftswort eine qualitative Eigenschaft, das Zahlwort eine quantitative. Qualität und (quantität sind korrelative Begriffe.

Dass die Imperativformen des Zeitworts in ihrem syntaktischen Wert mit den Ausrufwörtern Berührungspunkte haben, kann man leicht einsehen. Allgemein bekannt ist auch, dass einzclne Imperativformen sich endgültig in die Kategorie der Ausrufwörter verschoben haben, z.B. las 'siehe!'. Airila aber geht offenbar darüber hinaus, wenn er an einer Stelle (S. 101) Iehrt, dass »die Verba in ihren Imperativformen ihre Ausrufwörter haben", oder wenn er in der Gesamttabelle der semantischen Wortarten und der Wortartenumwandlungen (S. 102) die Imperativform opi 'lerne' des Verbs oppia 'lernen' geradezu in die Rubrik der Ausrufwörter, der Interjektionen einträgt. Wohin würde as führen, wenn wir die verschiedenen Formen des Paradigmas eines Wortes ihrem syntaktischen Gebrauch gemäss bald in die eine, bald in die andere Wortartgruppe einreihen würden?!

\section{T. Mit dem Anspruch der S'ystematisierung}

11. Aarni Pentrilä veröffentlichte vor kurzem die erste deskriptive finnische Grammatik wissenschaftlichen Anspruchs ${ }^{1}$. Die wissenschaftliche Systematisierung, die Synthese des Wissenswerten über die Erscheinungen der Sprachstruktur stellten den Verfasser unallsweichbar vor die Aufgabe, die traditionellen crrammatischen Regeln und Kategorien nach der Rolle, die sie im Ganzen der Sprachstruktur spielen, neu zu werten. Diese Seuwertung berührte gerade den traditionellen Begriff des Wortes und die traditionellen Wortarten am gründlichsten.

Penttilä sieht, dass der Ausdruck Wort ("sana") sowohl in der Alltigssprache als auch in den grammatischen Untersu-

1 Aarni Penttila Suomen kielioppi. 1957. Vgl. Istráx Papp Über einige Lehren der deskriptiven finnischen Grammatik (ungarisch). NyK 61 s. $418-427$. 
chungen bisher in mehreren verschiedenen Bedeutungen gebraucht wurde. Er selbst hebt die wissenschaftliche Einhelligkeit hervor und unterscheidet drei Wortbegriffe: 1. das "Wortvorkommen» ("sane») bedeutet die realen, verständlichen Phoneme und Grapheme, also alle die ausgesprochenen Iautreihen und geschriebenen Buchstabenreihen, bei denen sich an das sinnliche Erlebnis (Gehör-, Seherlebnis) eine bestimmte Bedeutung anknüpft; 2. die "Wortform» ("sananmuoto") ist die Klasse der ähnlichen Wortvorkommen, deren Realisierungen die realen Phoneme und Grapheme sind; 3. das "Wort" ("Sana") ist die Klasse der als zusammengehörend empfundenen Wortformen. Z.B. sooft wir dic Lautreihe talossa 'im Hause' aussprechen oder schreiben, mit soviel "Wortvorkommen" haben wir es zu tun. Alle diese konkreten Wortrorkommen sind die Verwirklichungen der einzigen "Wortform" talossa; die Wortform talossa ist aber sinnlich nicht mehr wahrnehmbar, es ist bloss ein sprachliches Zeichen, das auf der Ebene des Bewusstseins mit einem bestimmten grammatischen Wert lebt: das Zeichen für 'Haus' und für das, was damit, bzw. 'darin' verbunden ist. Endlich gehört die Inessivform talossa 'im Hause' geradeso wie z.B. der Nominativ Sing. talo 'llaus' oder der Nominativ Plur. talot '(die) Häuser' zusammen. Sie sind alle verschiedene Wortformen des "Wortes" talo 'Haus'; das Wort talo ist aber bloss ein sprachliches Zeichen, das nur auf der Ebene des Bewusstseins lebt, einen bestimmten lexikalischen Wert hat (die Bedeutung 'Haus'), jedoch keine grammatische Beziehung (Subjekt, Adverbiale usw.) besitzt. Eine "Wortform" des "Wortes» talo ist also der Inessiv talossa, und dass der letztere in einem ausgesprochenen oder geschriebenen Satz (z.B. hän asuu uudessa talossa 'er wohnt in einem neuen Haus') auf die Ebene der Realität tritt, ist nichts anderes, als das "Wortvorkommen" der Wortform talossa des Wortes talo.

Penttilä macht darauf aufmerksam, dass man bei der wissenschaftlichen Untersuchung der Gruppen der Wörter, der Wortarten, die drei besonderen Wortbegriffe in Betracht zu ziehen hat. Der Hauptfehler der traditionellen Wortartenaufteilung besteht eben darin, dass man zwischen "Würtern", "Wortformen" und "Wortvorkommen" keinen Unterschied machte. 
Die Folge davon war, dass man ein Wort in eine bestimmte Wortart einreihte, aber oft feststellen musste, dass das betreffende Wort in diesem oder jenem Satzzusammenhang einen vom Gewöhnlichen abweichenden Wortartwert hat.

Diesen Folgewidrigkeiten der traditionellen Aufteilung kann man nach Penttiläs Meinung nur so abhelfen, dass man die Klassifikation der "Wortarten", der "Wortformenarten" und der "Wortvorkommensarten" besonders durchführt.

12. Nach Penttiläs System zerfallen die "Wörter" der finnischen Sprache auf Grund ihrer Flexionseigentümlichkeiten in drei Gruppen, d.h. nur drei "Wortarten" sind möglich: 1. Indeklinabilia oder Partikeln, z.B. ohitse '(an etwas) vorbei', koko 'ganz'; 2. deklinicrbare Wörter, die der Biegung der Nomina folgen, also Nomina, z.B. aamu 'Morgen', kotona 'zu Hause', ylhäällä 'oben'; 3. konjugierbare Wörter, die der Biegung der Zeitwörter folgen, d.i. Verba, z.B. astun 'ich trete', tunnen 'ich fühle'.

Diese morphologische Klassifikation finden wir auch schon in dem traditionellen Wortartensystem, u.a. in Setäläs Schulgrammatiken. In ein-zwei Punkten wich aber Penttilä wissentlich von der Wortartentradition ab. So rechnet er Wörter wie ulkona 'draussen', ylhäällä 'oben' usw., die eine klare Formenkonstruktion, aber ein unvollständiges Paradigma haben, gewöhnlich zu den Partikeln, in Penttiläs System kommen sie aber in die Gruppe der deklinierbaren Wörter. Ferner rechnen die traditionellen Systeme die Infinitive III, IV und $\mathrm{V}$ wie lukemassa 'im, beim Lesen', lukeminen 'das Lesen', (olin) kaatumaisillani 'ich wäre beinahe gefallen', und die Partizipien: lukeva 'lesend', luettava 'zu lesen', lukenut 'gelesen; einer, der gelesen hat', luettu 'gelesen, Gelesenes' zum Verbalparadigma, während Penttilä diese in die deverbale Nomenbildung verweist und im Verbalparadigma nur den Infinitiv I und II belässt, also Verbalnomina wie lukea 'lesen', lukeakseni 'damit ich lese(n kann)', lukiessa 'während des Lesens', lukien 'lesend'.

13. Die "Wortformenarten" sind nichts anderes als die verschiedenen Formen der Nomina und Verba. 
Im Bereich der Nomina gehören hierher die Kasus, im Brreich der Verba aber das Genus actirum und passivum, dir verschiedenen Formen der Person, der Zeit, des Modus, ferner die Verbalnominia. Zı den letzteren zählt Penttilä wie wir sahen - nur den Infinitiv I und II.

In Penttiläs System sucht die Wortartenuntersuchung der verschiedenen nominalen und verbalen Formen dip syntaktischen Rollen dieser Formen, bzw. ihres Vorkommens 'zu klären. während die formalen Eigentümlichkeiten derselben Wortformen in der Lehre der Wortflexion behandelt werden.

14. Die "Wortvorkommensarten", d.h. die kategoriellen Gruppen der Wortvorkommen, können nach Penttilä auf Grund zweier Gesichtspunkte systematisiert werden: der Bedeutung und der Syntax. Da sich ein Bedeutungserlebnis in Wahrheit mur an das reale, d.h. wirklich ausgesprochene oder geschriebent' Wort, d.i. also an ein "Wortvorkommen" knüpft, hielt jed" bisherige Aufteilung auf Grund der Bedeutung letzten Endes die Arten der "Wortvorkommen" vor Augen. Ähnlich steht es mit der Wortartenverwendung des syntaktischen Prinzips. Nicht das "Wort", nur das reale "Wortvorkommen" erscheint im Zusammenhang mit der Rede und übernimmt eine bestimmte Rolle im Satze. Daher gelangen wir bei der Anwendung des syntaktischen Prinzips nicht zu den Kategorien der "Wörter", sondern denen der "Wortvorkommen".

Nach Penttilä kann man die Wortrorkommensarten auf Grund der Bedeutung in folgendem Syrstem darstellen:

A. Nomina

1. Interjektionen

2. Lautmalende Wörter

3. Anredewörter

4. Befehle

5. Ausrufe

6. Fürwörter

7. Nennwörter
a. Gattungsnamen
b. Stoffnamen
c. Artnamen
d. Eigennamen 
e. abstrakte Wörter

f. Eigenschaftswörter

g. Zahlwörter

h. Situativa

B. Verba

C. Partikeln

1. inhaltsvolle

2. gradbezeichnende

3. modifizierende

Zu bemerken ist noch, dass unter den Nomina die Interjektionen und dir lautmalenden Wörter - - da ohne Bedeutung - sich scharf absondern ron denen mit Bedeutung, d.i. den Fürwörtern und den verschiedenen Nennwörtern. Die Anredewörter, Befehle und Ausrufe bilden einen Übergangr zwischen den Kategorien mit und denen ohne Bedeutung. Die Gruppe der Verba teilt Penttilä nach der Qualität der Handlung in essenzielle, faktitive, inchnative usw. rin, nach dem dspekt aber in irresultative (imperfektive) und resultative (perfektive).

Ausser dieser Einteilung hat Penttilä auch noch andere semasiologische Klassifikationen in Betracht gezogen. Bei den Nomina spricht er von teilbaren und unteilbaren, anderseits von bestimmten, unbestimmten und halbbestimmten Wortvorkommen. Nicht bloss auf dif Nomina bezogen behandelt er dieKategorien der autosemantischen und der synsemantischen Wörter.

Die syntaktischen Gruppen der Wortrorkommen gestalten sich in P'enttiläs System folgendermasse'n:

A. Nomina und Partikeln
1. Hauptwörter
2. Fürwörter
3. Eigenschaftswörter
4. Bestimmungswörter
5. Postpositionen und Präpositionen
6. Bindewörter

I3. Verba

1. intransitive Verba

2. transitive Verba

3. transitiv-intransitive Verba

4. Verbiden 
Unter einigen Arten der Nomina und Partikeln können die Hauptwörter formal kongruente Ergänzungen zu sich nehmen, nicht aber die Fürwörtrr und Eigenschaftswörter. Die Bestimmungswörter, Postpositionen (Präpositionen) und die Bindewörter aber können weder eine formal kongruente Ergänzung zu sich nehmen, noch können sie in der Rolle einer solchen Ergänzung auftreten. Zu den Verbiden rechnet Penttilä die Partizipien, die Infinitive III und IV, und andere, gelegentlich verbale Natur zeigende (ein Objekt regierende) Nomina.

15. Penttilä hat in seiner revolutionierend neuen Systematisierung der Wortarten nichts anderes getan, als die traditionellen drei Grundlagen der Aufteilung: den morphologischen, semasiologischen und syntaktischen Gesichtspunkt den drei besonderen Wortbegriffen zugeordnet. Er stellte fest, dass man die Kategorien der "Wörter» und der "Wortformen" morphologisch voneinander absondern kann, die "Wortvorkommen" aber teilweise semasiologisch, teilweise syntaktisch zu gruppieren sind.

Nach unserer Ansicht ist in der systematisierung der Wortarten Penttiläs Fauptverdienst, dass er auf die Wichtigkeit der Untersuchung des realen "Wortvorkommens", also des im Zusammenhang mit der Rede, im Satz vorkommenden Wortes bei der Feststellung der Wortartkategorie hinwies. Es ist wahrlich richtig, dass das "Wort» grundsätzlich immer mehrsinnig ist und dass sich nur an das reale "Wortvorkommen" ein bestimmtes Bedeutungserlebnis knüpft. Eben darum kann man auch dip Wortartbedeutung endgültig nur im Zusammenhang mit der realen Rede, in der konkreten Sprechsituation, im Satze feststellen. Wenn wir z.B. von Herkules' Heldentaten sprechen, dann ist das Wort (nach Penttilä: das "Wortvorkommen») Herkules ein Eigenname, während wir im Satze er ist ein wahrhaftiger Herkules es mit einem Gattungsnamen, bzw. Artnamen zu tun haben. Dasselbe gilt auch für die Wortartkategorien syntaktischer Art. Penttilä hat recht: nur im realen Satzzusammenhang kann man die tatsächliche syntaktische Rolle, bzw. die darauf ruhende Kategorie der Wortart (Penttilä: "Wortvorkommenart») entscheiden. In dem Satze z.B. haluan kaunïn 
puvun 'ich will ein schönes Kleid' ist das Wort kaunis (mit Penttilä gesprochen: das gegebene "Wortvorkommen" der "Wortform" kauniin des "Wortes" kaunis) ein Eigenschaftswort, da es als Attribut mit seinem attribuierten Wort formal kongruiert. Hingegen im Satze hän harrastaa kaikkea kaunista 'ihn interessiert alles Schöne' ist es Hauptwort, da es eine formal kongruierende attributive Ergänzung zu sich nimmt. Nach unserem Erachten aber genügt es, aus allem die Folgerung zu ziehen, dass zur Feststellung des Wortartcharakters der Wörter die Untersuchung der gelegentlichen Wortbedeutung und der gelegentlichen Rolle im Satze unumgänglich nötig ist. Wir halten es für überflüssig, ein neues Fachwort, das des "Wortvorkommens" einzuführen.

Betreffs der Penttiläschen "Wortformarten" können wir dieselben vollständig weglassen, da eine so breite Auslegung der Wortarten, wonach jede Wortform eine besondere Wortart vertreten soll, den Rahmen des grammatischen Systems sprengen würde. Auch ist es nicht uninteressant, darauf hinzuweisen, dass Penttiläs "Wortarten" selbst sich auch nur aus der Untersuchung der entsprechenden "Wortvorkommen", d.i. aus der konkreten Sprechsituation ergeben. Von einem aus dem Redezusammenhang herausgehobenen "Wort" kann man ja nicht immer eindeutig feststellen, ob es der Deklination eines Nomens oder der Konjugation eines Verbs folgt, bzw. ob es flexibel oder unflexibel ist. Denken wir nur an solche Nomenverba wie z.B. ung. les. Nur dic Stellung im Satze zeigt, dass es dekliniert, also ein Nomen ist (z.B. lesben áll 'er steht auf der Lauer'), oder aber zum Paradigma einer Konjugation gehört, also ein Zeitwort ist (z.B. vadra les 'er lauert auf das Wild'). So können wir auch vom ung. Worte vagy an sich nicht wissen, ob es flexibel oder unflexibel ist.. Das kann nur seine Erscheinung im Satze klären. Im Satze szemtelen vagy 'du bist unverschämt' handelt es sich offenbar um ein Verb, da hier vagy 'du bist' das Mitglied des Verbalparadigmas ragyok 'ich bin', vagy 'du bist', van 'er ist' usw. ist. In der Redensart vagy Napóleon, vagy kalaposinas 'entweder Napoleon, oder ein Hutmacherjunge' ist ragy Bindewort, da es zu keinem Paradigma gehört. Penttiläs "Wortarten" sind also in Wirklichkeit "Wortvorkommen- 
arten", und schon darum ist es nicht zweckmässig, Penttiläs Terminologie in der Analyse der Wortarten in Gebrauch zu nehmen.

Wir müssen uns dessen bewusst sein, dass bei der Untersuchung der Wortarten auch die Anwendung des morphologischen und syntaktischen Gesichtspunkts Gefahren birgt. Es kann nämlich vorkommen, dass jemand die Wortform bzw. die syntaktische Rolle mit der Kategorie der Wortart identifiziert. Diesen Fehler begann -- wie wir sehen -- Penttilä damals, als ar die verschiedenen Nominalformen (z.B. den Inessiv lalossa 'im Hause') und die einzelnen Verbalformen (z.B. die Form Akt. Kond. Präs. 1. P. Sg. p!y!täisin “ich würde bitten`) als besondere Wortarten ("Wortformenarten") "qualifizierte, oder als er Aurufe (z.B. Kalle! 'Karl'), Befehle (z.B. kädet ylös! 'Hände auf'), Ausrufo (z.B. suurenmoista! 'grossartig') als "Wortvorkommenarten" behandelte, obwohl im ersteren Fall von den Formen der Wörter und nicht ron deren Gruppen die Rede ist; im letzteren Fall aber haben wir es mit Satzarten, nicht mit Wortarten zu tun. Solche Gefahren zu vermeiden gibt es eine sichere Methode: man muss die Wörter aus dem Satze herausheben, ihnen alle akzessorischen Beziehungselemente (Endungen, Zeichen) nehmen und muss das so erhaltene Wort selbst der Wortart nach werten. Nur wenn in der Bestimmunr des Wortartcharakters Zweifel oder I'nsicherheiten auftauehen, dann muss man das Wort wieder in seinen Satzzusammenhang zurückversetzen und nun anf Grund des realen Bedeutungsinhalts, der okkasionellen Formenstruktur und des syntaktischen Verhaltens den Wert der Wortart zu klären trachten. Sach der Heraushebung können die realen Akzessorien (Bedeutungsinhalt, morphologische Struktur, syntaktisches Verhalten) des zurückversetzten Wortes nicht mehr Eigenziel werden, sondern wahrlich nur Mittel bei jenem abstrahierenden Verfahren, als dessen Endergebnis wir zur Wortartenkategorie selbst gelangen künnen.

Panttilä hat einesteils Noreens, anderseits auch die Ergebnisse der vorhergehenden finnischen Forschung, besonders Ahlmans und Airilas in wrossem Masse benutzt. Einen ganz neuen Weg schlug er ein, indem a einen Teil der Verbalnomina - auf den Bereich der Wortbildung weisend -- aus dem Ver- 
balparadigma heraushob und nur die Infinitive I und II an ihren alten Stellen beliess. In Verbindung mit den von syntaktischen Gesichtspunkten aus betrachteten Klassen des Verbs spricht er über die Kategorie der "Verbiden". Verbiden nennt er diejenigen Nomina, die syntaktisch verbale Natur besitzen: sie können eine Objektsergänzung haben. Hier reiht Penttilä die Infinitive III und IV und die zwei Partizipien ein. Es wäre freilich folgerichtiger gewesen, auch die Infinitive I und II (und den ausgelassenen Infinitiv V) zur Kategorie der "Verbiden" zu rechnen, sind das doch auch Nomina, die ein Objekt zu sich nehmen können. Tatsache ist aber, dass die "Verbiden", oder mit ihrem alten Namen die "Verbalnomina» weder zur Gruppe der Verba, noch zu der der Nomina gehören. Das Verbalnomen ist eine selbständige Wortartkategorie, die den I'bergang zwischen Nomen und Verb bezeichnet ${ }^{1}$.

Das Streben nach Systematisierung unterbleibt in Penttiläs Wortartenuntersuchungen auch sonst an mancher Stelle. So zieht er in Verbindung mit den semantischen Gruppen der "Wortrorkommen" mehrere Klassifikationen in Betracht, ohme zu versuchen, die verschiedenen Klassifikationen miteinander in Einklangr zu bringen. Dieses Verfahren ist bis zu einem gewisseu Grade verständlich; hat doch den Verfasser bei seinem Systematisierungsbestreben in seiner Arbeit auch das Ziel geführt, keine wesentliche Spracherscheinung aus seiner Grammatik auszulassen - selbst dann nicht, wenn es ihm vorläufig auch nicht gulang, deren Stelle im system in ausreichendœr Weise zu klären.

\section{I'I. Die Lehren der Sprachgeschichte}

16. Lauri Hakulines hat in seinem Buch über die Struktur und Entwicklung der finnischen Sprache der Frage der Wortarten ein besonderes Kapitel gewidmet ${ }^{2}$.

1 Vgl. Istrax Papp Infinittimuotojen asema kieliopissa. Verba docent. Juhlakirja Lauri Hakulisen 60-vuotispäiväksi 6. 10.1959. S. $255-268$.

2 Ladri Hakclixex Suomen kielen rakenne ja kehitys I. 1941. S. $61-72$; dasselbe russisch: Развитие и структура финского языка I. 
Er geht davon aus, dass auf der Stufe des primitiven Sprachzustandes zwischen Wort und Satz wohl kein Unterschied bestand. Der sprachliche Ausdruck des Gedankens, der Satz, scheint grundsätzlich älter zu sein als das Wort, doch praktisch war der uralte Satz wohl eingliedrig und fiel mit dem Wort zusammen. So konnte z.B. das Wort kala als Satz soviel wie 'ich will Fische essen' oder 'fang diesen Fisch!' oder 'ich habe den Fisch gefangen' bedeuten. Dieses satzwertige kala konnte aber weder in seinem Bedeutungsinhalt noch in seinem morphologischen und syntaktischen Werte mit dem hentigen begriffbedeutenden Wort kala 'Fisch' nominativischer Form und Rolle identisch sein. Unsere Begriffe sind die Ergebnisse unserer abstrahierenden 'Tätigkeit. Das menschliche Denken konnte sich nur nach langer Entwicklung aus der Welt der konkreten Dinge zu solchen Begriffen emporheben wie 'Fisch'. Das Satzwort kala der Ursprache konnte weder vom morphologischen noch vom syntaktischen Gesichtspunkte mit dem späteren, im heutigen Sinn verstandenen Nominativ lala identisch gewesen sein; bestand doch im Anfang nur eine Wortform, während dazı. dass wir irgendeine Form als Nominativ verstehen sollen, es nötig ist, dass auch andere nicht-nominativische Formen existierten. Die verschiedenen Flexionskategorien haben sich nach dem Grundsatz des Gegensatzes ausgebildet; geradeso können wir uns die Ausbildung der Wortartenkategorien auf der Grundlage der Gegensätzlichkeit, der Polarisierung vorstellen. Es konnte keine Nomina geben, solange es keine Nicht-Nomina, d.i. keine Verba gab, und umgekehrt. Die Wortkategorien sonderten sich aus dem ungegliederten, mit mehreren Funktionen belasteten Satz ab, so dass die einzelnen Wörter sich langsam für besondere Rollen spezialisierten.

17. Die finnische Sprache hat bis heute Andenken an jene Zeit bewahrt, in der die Wortarten sich noch nicht klar voneinander unterschieden. Bewahrte Altertümlichkeiten solcher Art sind jene verbalen Wurzelwörter, deren Stamm auch als Nomen fungiert, z.B. sulaa 'schmilzt' $\sim$ sula 'geschmolzen,

Москва 1953. S. 63-72; deutsch: Handbuch der finnischen Sprache I. Wiesbaden 1957. S. 44-52. 
flüssig', sylkee 'spuckt' sylki 'Speichel', tuulee 'bläst' tuuli 'Wind'. Die finnischen Präsensformen wie tekee gehen über eine Form tekevi auf eine Lautform tekevä zurück, und letzteres lebt heute als Nominativ Sg. des aktiven Partizipiums I weiter. Tekevät und ähnliche pluralische Formen haben auch heute zwei Funktionen: als finite Verbalformen ('sie tun'), anderseits als Partizipien ('tuende'). Auf die einstige Gemeinschaft der Nomina und Verba deutet auch der Umstand, dass z.B. das Suffix -va/-vä nicht nur an Verbalstämme tritt (tekee 'er tut': tekevä 'tuend'), sondern auch an Nominalstämme (liha 'Fleisch': lihava 'fleischig, fett').

Anch zwischen den einzelnen Nomenarten bestand einst kein so bestimmter Unterschied wic heute. Das Eigenschaftswort z.B. sonderte sich ziemlich spät vom Hauptwort ab. Darauf deutet, dass - abgesehen von der nachträglichen Substantivierung des Eigenschaftswortes (z.13. nuori 'jung' $\rightarrow$ 'junger Mann') - es solche Wörter gibt, bei denen der substantivische und adjektivische Gebrauch gleich alt ist, z.B. kylmä 'kalt, Kälte', vilu 'Frost, frostig', hämäräa 'Dunkel, dunkel'. Hierher gehört auch, dass bei als Substantiv gebrauchten Adjektiven das regierte Substantiv im Partitiv steht, z.B. pitkän matkaa 'einen langen Weg', lyhyelisi aikaa 'auf kurze Zeit', viimeisellä kertaa 'zum letzten Male', oder dass man ein Substantiv manchmal wie ein Adjektiv steigern kinn, z.B. perä 'der Hintere': peremmäksi 'weiter hinten', leerät 'Frühling': keväämpänä 'später im Frühling'. Endlich gibt es Suffixe, mit denen man sowohl Substantiva als Adjektiva bilden kann, z.B. nuorukainen 'junger Mann' ～jäinen 'eisig', isäntä 'Wirt' vihanta 'grünend'.

Die finnischen Zahlwörter entwickelten sich aus Substantiven, die' 'Gruppe, Schar' bedeuteten. Heute haben sie ihren ursprünglichen substantivischen Charakter nur noch im Nominativ und Akkusativ der Einzahl bewahrt (z.B. kymmenen miestä 'zehn Männer', genauer: 'zehn von den Männern'), in den casus obliqui wurden sie zu Adjektiven (z.B. kymmenelle miehelle 'zehn Männern'). Ferner gibt es Zahlwörter, die in bestimmten Wendungen auch heute ganz als Hauptwörter gebraucht werden, z.B. vuosikymmen 'Jahrzehnt' (wörtlich: 'Jahrzehn'), vuosisata 'Jahrhundert'. 
Viele Zeichen weisen auf das uralte Wesen der Wortart der Pronomina hill; so der Umstind, dass mehrere gerade uralischen Ursprungs sind. Ein Zeichen des uralten Wesens der Pronominaldeklination sind die rielfach festzustellenden Unregelmässigkeiten, z.B. die besondere Pluralisierung in solchen Beispielen wie tämä 'dieser': nämä 'diese', two 'jener': nuo 'jene', se 'der': ne 'die'. Die persönlichen Fürwörter sind auf jeden Fall älter als die bestimmten Formen des Verbs, da die verbalen Personalendungen sich aus den entsprechenden persönlichen Fürwörtern entwickelten. Man hat bloss bei einem Fürwort dessen substantivischen Ursprung nachgewiesen: itse(nsä) 'selbst'.

Die Partikeln sind --- abgesehen ron den Interjektionen -alle neuen Ursprungs. So haben sich die Bestimmungswörter, die Postpositionen und Präpositionen entweder aus nominalen oder ans verbalen Paradigmen entwickelt - oder sie sind Entlehnungen aus fremden Sprachen. Unter den Bindewörtern gibt as keins finnisch-ugrischen Ursprungs, und soweit sie nicht fremde Entlehnungen sind, haben sie sich aus älteren Bestimmungswörtern entwickelt. So z.B. kun 'als, wenn' $\sim$ kuin 'wie, als' sind ursprünglich nichts anderes als Instruktivformen vom Pronominalstamm ku- mit der Bedeutung 'wie, auf welche Art'. Että 'dass' ist ein Derivat eines Pronominalstammes $e$ 'dieser', seine ursprüngliche Bedeutung war 'so'. Das Bindewort jos 'wenn' ist der Lativ des Pronominalstammes jo-, mit der Bedeutung 'darauf, auf jene Art'. Das Bindewort $t a(h) i$ 'oder' hängt mit Verb tahtoa 'wollen' zusammen.

18. Hakulinen zeigt uns in seinem Buche die Struktur und die Entwicklung der finnischen Sprache in ihrer Einheit auf und lässt uns empfinden, dass die strukturalen Eigentümlichkeiten und die Entwicklungstendenzen eng aufeinander angewiesen sind: darin liegt der grosse Wert seiner Arbeit. Er weist im Kapitel über Wortarten auf solche Tatsachen hin, die zwar Eigentümlichkeiten des synchronischen Sprachsystems, aber gleichzeitig auch historisch verwertbar sind, wie z.B. die Nomenverba oder die Erscheinung des Substantiv-Adjektivs. Dadurch, dass er die bewahrten Altertümlichkeiten in den Vor- 
dergrund stellt, vertieft sich die Untersuchung des Wortartensystems zur Erforschung der Entwicklung der Wortarten.

Hakulinen hat den Weg der Entwicklung der Wortarten an Hand von klaren, überzeugenden Beispielen aufgezeigt. Die uralten Kategorien sind das Ergebnis gleichzeitiger Separation, die Separation selbst hingegen ist nach dem Grundsatz des Gegensatzes vor sich gegangen. Die Differenzierung der Formen voneinander ist wahrhaft eine so grundlegende Gesetzmässigkeit, die sich auf jeder Linie der Sprachentwicklung offenbart. Doch würde es aber nicht schaden, neben der Tendenz der gegensätzlichen Separation auch diarauf hinzuweisen, dass wir auch für Integrierung der einmal so ausgebildeten gegensätzlichen Wortkategorien bzw. für die synthese Beispiele haben. Wir sehen die Sache so, dass auf dem Wege der gleichzeitigen Separation des uralten Nomenverbs das Nomen und das Verb, diese zwei gegensätzlichen Wortartenkategorien, zustandegekommen sind. Nichdem sich num das Nomen und das Verb schon entwickelt hatten, war die Möglichkeit für den Integrationsprozess gegeben: eine synthese der verbalen und der nominalen Eigentümlichkeiten hat stattgefunden, indem die Kategorie des Verbilnomens ins Leben getreten ist ${ }^{\mathbf{1}}$.

\section{Las Wort in der Siprachergleichung}

19. Paavo Ravila hat im Anschlusse an seine Untersuchung über die Eigentümlichkeiten des uralischen Satzes mehrmals Fragen der Wortarten behandelt ${ }^{2}$. Er untersuchte das Problem der Wortarten auch in ciner besonderen Studie, in der er besonders die uralischen sprachen in Betracht zog ${ }^{3}$.

1 Vgl. Istráx PAPP Infinittimuotojen asema kieliopissa. A.a.O.

2 Vgl. Paavo Ravila Ïber die Verwendung der Numeruszeichen in den uralischen Sprachen. FUF 27 S. 119 ff.; Uralilaisen lauseen alkuperäisestä rakenteesta. Vir. 1943 s. 258 ff.; Lauseopin periaatekysymyksiä. Vir. 1944 S. 110-111; Johdatus kielihistoriaan. 1946. S. 64-65.

3 PaAvo Ravila Sanaluokat, erityisesti uralilaisia kieliä silmällä pitäen. Vir. 1953 S. 41-50; dasselbe deutsch: Die Wortklassen mit besonderer Berücksichtigung der uralischen Sprachen. JSFOu. 59. Vgl. 
Ravila vertritt in der Erforschung der Wortarten die historische Anschauung. Nach ihm ist jede grammatische Kategorie, auch die Wortarten, das Ergebnis historischer Entwicklung. Das ist die Ursache davon, dass man kein ganz konsequentes und geschlossenes Wortartensystem aufbauen kann. Bei jeder Klassifikation der Wortarten können drei Gesichtspunkte in Betracht kommen: 1. die Bedeutung des Wortes, 2. die Rolle des Wortes im Satz, 3. die Flexionsverhältnisse des Wortes. Beim semantischen Gesichtspunkt ist die Hauptschwierigkeit, dass viele Hauptwörter eine Handlung oder eine Eigenschaft bedeuten. So bedeuten z.B. hyvä 'gut' und hyvyys 'Güte' beide eine Eigenschaft, obwohl das eine Eigenschaftswort oder Hauptwort das andere Hauptwort ist. Auf die syntaktische Rolle kann man nicht bauen, da manchmal auch ein ganzer Satz in der Rolle eines Wortes stehen kann. Z.B. näin pojan 'ich sah einen Knaben' und näin, että poika tulee 'ich sah, dass ein Knabe kam (eigentl. kommt)', in diesen Sätzen steht ein Hauptwort bzw. ein besonderer Satz als Objekt. Verhältnismässig am klarsten ist der dritte Gesichtspunkt. Unter Beachtung der Flexionsverhältnisse kann man die Wörter in Nomina, Verba und Partikeln einteilen. Aber auch diese Einteilung ist nicht befriedigend. Besonders die Verbalnomina verursachen Schwierigkeiten: diese werden nämlich wie Nomina flektiert, nach ihrem Rectum beurteilt haben sie aber verbale Natur, z.B. tulitikkuja lainaamassa 'beim Leihen von Zündhölzchen'. Darum kehrt Ravila im Gegensatz zu jedem neueren Bemühen zu der Ansicht zurück, dass man alle drei Gesichtspunkte zusammen in Betracht zu ziehen hat. Das hat freilich zur Folge, dass man so kein allgemeines, für jede Sprache in gleichem Masse gültiges System aufbauen kann. Was man z.B. in verschiedenen Sprachen Nomen nennt, bezeichnet nicht immer dieselbe Erscheinung. Im Fimnischen ist z.B. die besondere Konjugation das wichtigste Merkmal des Verbs. Im Mordwinischen hingegen kann man auch das Nomen so flektieren wie ein Verb, z.B. erzáan 'ich bin ein Erza', erzáat 'du bist ein Erza',

Istvan Papp Az ósi szófajok. Pais-Emlékkönyv (Die uralten Wortarten. Festschrift für Pais). 1956. S. 29-35. 
erza 'er ist ein Erza'. Dasselbe liegt auch im Samojedischen vor, doch sind darum auch zwischen den einzelnen samojedischen Sprachen Unterschiede. Im Juraksamojedischen kann das Nomen auch ein Zeitverhältnis bezeichnen, im Ostjaksamojedischen nicht. Wenn wir daher in der mordwinischen Grammatik den Terminus "Nomen» gebrauchen, wollen wir damit nur soviel aussagen, dass die "Nomina" genannten Wörter der mordwinischen Sprache annähernd den in den europäischen Sprachen unter demselben Namen bekannten Wörtern entsprechen. Diese Terminologie entspricht praktischen Ansprüchen vollständig, von wissenschaftlichem Standpunkt ist sie auch nicht zu beanstanden, wenn wir zugleich die besonderen Eigentümlichkeiten des mordwinischen Nomens aufzählen.

Unzweifelhaft war das Wortartensystem der finnisch-ugrischen Grundsprache ein anderes als das der heutigen finnischugrischen Sprachen. Das Postpositionensystem der finnischugrischen Sprachen ist z.B. überwiegend eine neue Eigentümlichkeit. Auch die Kategorie des Bestimmungswortes können wir kaum als sehr alt betrachten. Auch können wir Parallelentwicklungen in einzelnen verwandten Sprachen bemerken. Der Grund ist, dass das von der Grundsprache ererbte Sprachsystem an mehreren Orten, unabhängig voneinander zum selben Ergebnis führte. Wenn also eine grammatische Kategorie auch in allen finnisch-ugrischen Sprachen zu finden ist, bedeutet noch nicht, dass wir es mit einer gemeinsamen, grundsprachlichen Erbschaft zu tun haben.

20. Wir wissen seit langem, dass die nominalen und verbalen Stämme der uralischen Grundsprache zweisilbig waren und keinen Unterschied in der Lautstruktur untereinander aufwiesen. Ein ganz anderes Bild zeigen die Pronomina, denen sich auch das negierende Hilfszeitwort anreiht: sie waren ursprünglich einsilbig. Vom rein formalen Standpunkt sonderten sich die Wörter der uralischen Grundsprache also in zwei Gruppen: einsilbige und zweisilbige. Bemerkenswerterweise stimmt mit dieser rein formalen Gruppierung auch die Teilung nach der Bedeutung überein: die Zeigewörter und zugleich die Ausrufwörter waren einsilbig, die Nennwörter aber zweisilbig. Den 
lautlichen und semantischen Standpunkt zusammenfassend können wir also fürs Uralische zwei proethnische Kategorien annehmen: I. einsilbige Zeige- und Ausrufwörter. II. zweisilbige Nennwörter. Chronologisch können wir sagen, dass die Ausbildung dieser beiden uralten Wortarten in der Zeit vor der uralischen Grundsprache, also in der präuralischen Periode vor sich gegangen ist.

Im Laufe der weiteren Entwicklung zerfiel die Kategorie der Nennwörter in Nomina und Verba. Diese Teilung hat zur Zeit der uralischen Grundsprache begonnen. Allgemein ist man der Ansicht, dass in den uralischen Sprachen die Kategorie des Nomens älter sei als die des Zeitworts. Ravila aber schliesst sich in dieser Frage Hakulinen an: die auch als Nomina gebrauchten Verbalstämme (sula-, sylke-, tuule- usw.) sind bewahrte Altertümlichkeiten aus jener Zeit, in der die Grenzen zwischen den Wortarten noch nicht ausgebildet waren.

Zur Aufklärung des Absonderungsvoruangs der Nomina und Verba liefert die Klärung der ursprünglichen Funktion der Nominal- und Verbalstämme interessante Angaben. Im Finnischen und in mehreren anderen finnisch-ugrischen Sprichen ist der Verbalstamm heute eine reine Abstraktion, da derselbe im Verbalparadigma keine selbständige Funktion hat. Anders steht die Sache bei den Nominalstämmen: in den uralischen Sprachen lebt der Nominalstamm heute als Nom. Sg. weiter.

Es gibt aber auch einige uralische Sprachen, in denen auch der reine Verbalstamm realen Wert hat: in der Funktion als Präsens oder Aorist Einzahl 3. Person. Im Samojedischen z.B. werden die Aoristformen der subjektiven Konjugation so gebildet: hadam 'ich töte', hadan 'du tötest', hada 'er tötet' usw. Hier kann man die ursprüngliche Bedeutung des Stammes mit einem den Agens bezeichnenden Nomen ausdrücken: hadam 'ich (bin) Töter', hadan 'dı (bist) Töter', hada 'er (ist) Töter'. In den entsprechenden Formen der objektiven Konjugation hingegen - hadau 'ich töte es', hadar 'du tötest es', hadada 'er tötet es' -. kann man die ursprüngliche Funktion des Verbalstammes mit der eines Handlungsnomens identifizieren: hadau 'mein Töten', hadar 'dein Töten', hadada 'sein Töten'. Die alteste Funktion des Verbalstammes aber wird wohl ein Nomen 
gewesen sein, das eine Handlung bezeichnete, da sich aus dieser die Stammbedeutung der subjektiven und objektiven Flexionsreihe gleichmässig entwickeln konnte. Die subjektive Funktion mochte sich dann aus derartigen Bedeutungsgebilden entwickeln wie: 'Töten-ich', die objektive hingegen aus: 'Tötenmeines, mein Töten'. Dass dann die Stammbedeutung in der subjektiven Konjugration sich veränderte, zu einem Nomen agentis wurde, darin hat der bedentungsverengende Einfluss der parallel bestehenden objektiven Verbalformen keinen geringen Anteil. In solchen finnisch-ugrischen Sprachen. in denen nur eine einheitliche Konjugation geblieben ist, haben verschiedene akzessorische Elemente cine solche Verengung der Stammbedeutung durchgeführt, z.B. finn. juoksee 'er läuft' < juokseva, wo das Formans - $2 a-r a ̈$ zum Stamm juokse- trat.

In jener Zeit also, in der die Nomina und die Verba sich noch nicht voneinander absonderten, konnte der reine Wortstamm -. mit der Bedeutung eines Handlungsnomens - einerseits nominativische Funktion haben, anderseits Einzahl 3. Person sein. Das war die Urzelle jeder späteren Nominalform und der Verbalformen. Die Absonderung der nominalen und verbalen Flexion, zugleich die Separierung der nominalen und verbalen Wortkategorien konnte dann im zweigliedrigen Satz vor sich gehen.

21. Die Bedeutung ron Ravilas Wortartenstudie sehen wir darin, dass er abweichend von jedem einseitigen, auf einen Gesichtspunkt eingestellten Klassifizierungsversuch die gleichzeitige Berücksichtigung der drei traditionellen Gesichtspunkte und die Anwendung der historischen Betrachtungsweise betonte. Seine Ergebnisse scheinen fest zu sein. Demnach hat die voruralische Sprache zwei Wortarten ausgebildet: die einsilbigen Pronomina und Ausrufwörter, ferner die zweisilbigen Nennwörter. In der uralischen Grundsprache begann die Absonderung der Nennwörter in Nomina und Verba. Unserseits können wir hinzufügen, dass die Gruppe der voruralischen einsilbigen, subjektiv gefärbten Wörter ursprünglich einheitlich gewesen sein wird und sich erst zur Zeit der uralischen Grundsprache in zwei Wortartenklassen abzusondern begann: einerseits in Aus- 
rufwörter, anderseits in Demonstrativwörter. In der uralischen Grundsprache konnte es demgemäss nur erst die folgenden vier Wortarten geben: 1. Ausrufwörter, 2. Pronomina, 3. Nomina und 4. Verba. Jede andere Wortartgruppe musste sich erst nach der Zeit der uralischen Grundsprache ausgebildet haben. Wie das vor sich ging, haben die Erforscher der einzelnen uralischen Sprachen klarzulegen.

Die Schlusslehre ist: wenn e's auch für die Ausbildung der Wortarten und für ihre Verdichtung zl cinem System allgemeine Bewusstseinsbedingungen und grosse, in jeder Sprache zu findende Richtlinien gibt, ging die Ausbildung und Systematisierung der Wortarten auf verschiedenen Wegen in Raum und Zeit vor sich. Die Darstellung der Grundlinien einer wissenschaftlich wertvollen allgemeinen Wortartentheorie wird am sichersten auf induktivem Weg geschehen können: auf Grund der Lehren, die sich aus der Ausbildung der Wortarten und der Wortartensysteme in den einzelnen Sprachen und sprachfamilien ergeben.

Is'TVÁN PaPp 